\title{
SOME EXAMPLES OF NONASSOCIATIVE COALGEBRAS AND SUPERCOALGEBRAS
}

\author{
Daniyar Kozybaev ${ }^{1}$, Ualbai Umirbaev ${ }^{2}$, and Viktor Zhelyabin ${ }^{3}$,
}

\begin{abstract}
Locally finiteness of some varieties of nonassociative coalgebras is studied and the Gelfand-Dorfman construction for Novikov coalgebras and the Kantor construction for Jordan super-coalgebras are given. We give examples of a non-locally finite differential coalgebra, Novikov coalgebra, Lie coalgebra, Jordan super-coalgebra, and right-alternative coalgebra. The dual algebra of each of these examples satisfies very strong additional identities. We also constructed examples of an infinite dimensional simple differential coalgebra, Novikov coalgebra, Lie coalgebra, and Jordan super-coalgebra over a field of characteristic zero.
\end{abstract}

Mathematics Subject Classification (2020): 17B62, 17C70, 17D25, 17D15.

Key words: coalgebra, coderivation, differential algebra, Novikov algebra, Lie algebra, Jordan superalgebra, right-alternative algebra.

\section{INTRODUCTION}

The notion of coalgebra is dual to the notion of algebra. The theory of (co)associative coalgebras has been developed for a long time within the framework of the theory of Hopf algebras [18]. The study of coalgebras, bialgebras, and Hopf algebras received a new impetus when the term "quantum group", along with revolutionary new examples, was launched by V. Drinfeld in 1986 [3]. Lie bialgebras, which are simultaneously Lie algebras and Lie coalgebras, were introduced as one of the most important notions of quantum group theory [4]. The study of Lie coalgebras, investigated earlier by W. Michaelis [11], was intensified. It is well known that the dual of an associative coalgebra is an associative algebra and the dual of a Lie coalgebra is a Lie algebra. In 1994 J. Anquella, T. Cortes, and F. Montaner [1] called a coalgebra $C$ an $\mathfrak{M}$-coalgebra if the dual algebra $C^{*}$ belongs to the variety of algebras $\mathfrak{M}$. This allows to define alternative, Jordan, Malcev, left-symmetric, Novikov coalgebras, and so on.

The Fundamental Theorem on Coalgebras asserts that every finitely generated associative coalgebra over a field is finite-dimensional. An analogue of this result is true for alternative and Jordan coalgebras [1], for structurable coalgebras [20], for Jordan copairs [24], for right alternative Malcev admissible coalgebras and binary $(-1,1)$-coalgebras [15].

\footnotetext{
${ }^{1}$ Department of Mathematics, Eurasian National University, Nur-Sultan, Kazakhstan, email:kozybayev@gmail.com

${ }^{2}$ Department of Mathematics, Wayne State University, Detroit, MI 48202, USA; Department of Mathematics, Al-Farabi Kazakh National University, Almaty, 050040, Kazakhstan; and Institute of Mathematics and Mathematical Modeling, Almaty, 050010, Kazakhstan, e-mail: umirbaev@wayne.edu

${ }^{3}$ Institute of Mathematics of the SB of RAS, Novosibirsk, 630090, Russia, e-mail: vicnic@math.nsc.ru 
Lie coalgebras are not locally finite and the first example of an infinite dimensional finitely generated Lie coalgebra was given in [11]. In 1995 A. Slinko [17] found some necessary and sufficient conditions for Lie coalgebras to be locally finite. A connection between Jordan and Lie (super)coalgebras, which is an analog of the well known Kantor-Koecher-Tits construction for usual (super)algebras, was found in [21, 22]. M.E. Goncharov and V.N. Zhelyabin [6, 7] showed that every Malcev coalgebra embeds into a Lie coalgebra with triality. Unlike Jordan coalgebras, Jordan super-coalgebras are not locally finite [22].

In 2000 D. Kozybaev [9] constructed an example of a non-locally finite right-symmetric coalgebra and an example of a non-locally finite right-alternative coalgebra. I. Shestakov reported [16] that the example of a right-alternative coalgebra given in [9] is incorrect. This report attracted the attention of the authors to these old examples. First of all we noticed that the left-symmetric analogue of the non-locally finite right-symmetric coalgebra from [9] is a Novikov coalgebra. Moreover, we noticed that the commutator coalgebra of this coalgebra is exactly the non-locally finite Lie coalgebra given by $\mathrm{W}$. Michaelis [11]. In order to understand the nature of these examples we started to study codifferential coalgebras.

To any associative and commutative differential algebra $A$ one relates the following three algebras:

(1) a Novikov algebra obtained from $A$ by the Gelfand-Dorfman construction;

(2) a Lie algebra obtained as the commutator algebra of the Novikov algebra mentioned in (1); and

(3) a Jordan superalgebra obtained from $A$ by the Kantor construction.

The notion of coderivation allows us to define the notion of a (co)differential coalgebra. We constructed a very easy example $C$ of a non-locally finite associative and commutative differential coalgebra. Using this example we constructed three examples of non-locally finite coalgebras. We define an analogue of the Gelfand-Dorfman construction for coalgebras and using this we give an example of a non-locally finite Novikov coalgebra obtained from $C$, and the dual algebra of this coalgebra satisfies the identity $(x y) z=0$. This example coincides with Kozybaev's example mentioned above. Moreover, the commutator coalgebra of this Novikov coalgebra is exactly the non-locally finite Lie coalgebra given by Michaelis [11]. This Lie coalgebra is metabelian. Using $C$ and an analogue of the Kantor construction for super coalgebras, we give an example of a non-locally finite Jordan supercoalgebra. The dual of this super-coalgebra satisfies the super identities $x y=y x, x z=z x$, and $\left(z z_{1}\right)\left(z_{2} z_{3}\right)=0$ for even variables $x, y$ and for odd variables $z, z_{1}, z_{2}, z_{3}$.

We repeated the same route starting from the simple differential algebra $\left(F[x], \partial=\frac{d}{d x}\right)$ over a field $F$ of characteristic zero. The graded dual of this algebra is a simple infinite dimensional differential coalgebra. Recall that a coalgebra is called simple if it does not have any proper subcoalgebras. Applying the Gelfand-Dorfman construction to this coalgebra, we get an example of a simple infinite dimensional Novikov coalgebra. This coalgebra is the graded dual of the Novikov-Witt algebra $\mathcal{L}_{1}$ [10]. The commutator Lie coalgebra of this coalgebra is also simple and coincides with the graded dual of the Witt algebra $W_{1}$. And finally, using the Kantor construction, we constructed an example of a simple infinite dimensional Jordan super-coalgebra. 
We also noticed that the example of a non-locally finite right-alternative coalgebra from [9] can be fixed only by interchanging the indexes $3 n-2$ and $3 n-1$ on lines 3 and 4 of the formula (12). The fixed example is given in Section 6. A much more complicated example of a non-locally finite right-alternative coalgebra was recently given in [16].

The paper is organized as follows. In Section 2 we give some necessary terminology of coalgebras, notations, and general statements. In Section 3 we give examples of non-locally finite differential, Novikov, and Lie coalgebras. Simple infinite dimensional differential, Novikov, and Lie coalgebras are given in Section 4. Section 5 is devoted to Jordan supercoalgebras and Section 6 is devoted to right-alternative coalgebras.

\section{CoAlgebras And CODERIVATions}

Let $F$ be an arbitrary field. For any vector space $V$ over $F$ denote by

$$
V^{*}=\operatorname{Hom}_{F}(V, F)
$$

its dual vector space, i.e., the vector space of all linear forms on $V$.

Denote by

$$
V^{\otimes n}=\underbrace{V \otimes \ldots \otimes V}_{n}
$$

the $n$-th tensor power of the vector space $V$ over $F$.

The map

$$
\rho:\left(V^{*}\right)^{\otimes n} \rightarrow\left(V^{\otimes n}\right)^{*}
$$

defined by

$$
\rho\left(f_{1} \otimes \ldots \otimes f_{n}\right)\left(\sum_{i_{1} \ldots i_{n}} e_{i_{1}} \otimes \ldots \otimes e_{i_{n}}\right)=\sum_{i_{1} \ldots i_{n}} f_{1}\left(e_{i_{1}}\right) \ldots f_{n}\left(e_{i_{n}}\right)
$$

is injective. For this reason we can assume that

$$
\left(V^{*}\right)^{\otimes n} \subseteq\left(V^{\otimes n}\right)^{*}
$$

If $\phi: V \rightarrow U$ is a linear map of vector spaces then the transpose $\phi^{*}: U^{*} \rightarrow V^{*}$ of $\phi$ is defined by

$$
\phi^{*}\left(u^{*}\right)(v)=u^{*}(\phi(v)), \quad v \in V, u^{*} \in U^{*} .
$$

A vector space $C$ over $F$ with a linear map

$$
\Delta: C \rightarrow C \otimes_{F} C
$$

is called a coalgebra. The map $\Delta$ is called its comultiplication. We often call the pair $(C, \Delta)$ a coalgebra in order to emphasize the comultiplication in question. For any $a \in C$, using the Sweedler notation (see [18]), we write

$$
\Delta(a)=\sum_{a} a_{(1)} \otimes a_{(2)} .
$$

If $C$ is a coalgebra, then

$$
(f g)(a)=\rho(f \otimes g)(\Delta(a))=\sum_{a} f\left(a_{(1)}\right) g\left(a_{(2)}\right), \quad f, g \in C^{*}, a \in C,
$$


defines a product on $C^{*}$ and this product turns $C^{*}$ into an algebra. Denote this product by

$$
m_{\Delta}: C^{*} \otimes C^{*} \rightarrow C^{*}
$$

The algebra $C^{*}$ or $\left(C^{*}, m_{\Delta}\right)$ is called the dual algebra of the coalgebra $(C, \Delta)$.

A coalgebra $(C, \Delta)$ is called coassociative if

$$
(\Delta \otimes \mathrm{id}-\mathrm{id} \otimes \Delta) \Delta=0,
$$

i.e., for any $a \in C$ we have

$$
\sum_{a}\left(\Delta\left(a_{(1)}\right) \otimes a_{(2)}-a_{(1)} \otimes \Delta\left(a_{(2)}\right)=0 .\right.
$$

It is well known that a coalgebra $(C, \Delta)$ is coassociative if and only if its dual algebra $C^{*}$ is associative. Moreover, a coalgebra $(C, \Delta)$ is a Lie coalgebra if and only if its dual $C^{*}$ is a Lie algebra [11]. Following these results, the definition of coalgebras from any variety of algebras was given in [1]:

Let $\mathfrak{M}$ be an arbitrary variety of algebras. A coalgebra $(C, \Delta)$ is called an $\mathfrak{M}$-coalgebra if its dual algebra $C^{*}$ belongs to $\mathfrak{M}$.

Let $V$ be a vector space and let $\tau: V \otimes V \mapsto V \otimes V$ be the ordinary flip, i.e., a linear map with $\tau(x \otimes y)=y \otimes x$ for all $x, y \in V$.

A coalgebra $(C, \Delta)$ is called cocommutative if

$$
\Delta=\tau \Delta
$$

i.e.,

for any $a \in C$.

Let $(C, \Delta)$ be an arbitrary coalgebra. A subspace $B$ of $C$ is called subcoalgebra of the coalgebra $(C, \Delta)$ if $\Delta(B) \subseteq B \otimes B$.

A subcoalgebra $B$ of a coalgebra $(C, \Delta)$ is called proper if $B \neq\{0\}, C$. A coalgebra $(C, \Delta)$ without proper subcoalgebras is called simple [14].

It is well known [1] that $C$ admits a $C^{*}$-bimodule structure. The left and right actions of $C^{*}$ on $C$ are defined by

$$
\alpha \cdot a=\sum_{(a)} a_{(1)} \alpha\left(a_{(2)}\right), \quad a \cdot \alpha=\sum_{(a)} \alpha\left(a_{(1)}\right) a_{(2)}, \quad \alpha \in C^{*}, a \in C .
$$

Moreover, a vector subspace $B$ of a coalgebra $C$ is a subcoalgebra if and only if $B$ is a $C^{*}$-subbimodule of $C$. Therefore the intersection of any set of subcoalgebras of $C$ is again a subcoalgebra.

Let $S$ be a subset of a coalgebra $C$. The smallest subcoalgebra $\operatorname{Coalg}(S)$ of $C$ that contains $S$ is called the subcoalgebra generated by $S$. In other words, $\operatorname{Coalg}(S)$ is the $C^{*}$-subbimodule of $C$ generated by $S$. If $S$ is a finite set then $\operatorname{Coalg}(S)$ is called finitely generated.

A coalgebra $(C, \Delta)$ is called locally finite if every finitely generated subcoalgebra of $C$ is finite dimensional. 
A linear map $d: C \mapsto C$ is called a coderivation of the coalgebra $(C, \Delta)$ if

$$
\Delta d=(d \otimes i d+i d \otimes d) \Delta,
$$

i.e.,

$$
\Delta(d(a))=\sum_{a} d\left(a_{(1)}\right) \otimes a_{(2)}+a_{(1)} \otimes d\left(a_{(2)}\right), \quad a \in C .
$$

A triple $(C, \Delta, d)$ is called a (co)differential coalgebra if $(C, \Delta)$ is a coalgebra and $d$ is its coderivation. A subspace $B$ of a differential coalgebra $C$ is called a subcoalgebra if $B$ is a subcoalgebra of $(C, \Delta)$ and $d(B) \subseteq B$, i.e., $B$ is a codifferentially closed subcoalgebra.

Lemma 1. Let $d$ be a coderivation of the coalgebra $(C, \Delta)$. Then its transpose $d^{*}$ is a derivation of the dual algebra $C^{*}$, i.e.,

$$
d^{*}(f g)=d^{*}(f) g+f d^{*}(g), \quad f, g \in C^{*} .
$$

Proof. Let $f, g \in C^{*}, a \in C$. Then

$$
\begin{gathered}
\left(d^{*}(f g)\right)(a)=(f g)(d(a))=\rho(f \otimes g) \Delta(d(a))=\sum_{a} f\left(d\left(a_{(1)}\right)\right) g\left(a_{(2)}\right)+f\left(a_{(1)}\right) g\left(d\left(a_{(2)}\right)\right) \\
=\sum_{a}\left(d^{*}(f)\right)\left(a_{(1)}\right) g\left(a_{(2)}\right)+f\left(a_{(1)}\right)\left(d^{*}(g)\right)\left(a_{(2)}\right)=\left(d^{*}(f) g+f d^{*}(g)\right)(a),
\end{gathered}
$$

which proves the statement of the lemma.

Corollary 1. If $(C, \Delta, d)$ is an associative and commutative differential coalgebra then $\left(C^{*}, m_{\Delta}, d^{*}\right)$ is an associative and commutative differential algebra.

Let $A$ be an algebra over $F$ with the multiplication

$$
m: A \otimes A \rightarrow A,
$$

i.e., $m(a \otimes b)=a b$ for all $a, b \in A$. Let

$$
m^{*}: A^{*} \mapsto(A \otimes A)^{*}
$$

be the transpose of $m$. Unfortunately, the image $m^{*}\left(A^{*}\right)$ of $m^{*}$ does not always belong to $A^{*} \otimes A^{*} \equiv \rho\left(A^{*} \otimes A^{*}\right) \subseteq(A \otimes A)^{*}$. The structure of the dual coalgebra $\left(A^{\circ}, \Delta^{\circ}\right)$ is a little complicated (see $[1,12])$.

Let

$$
A=\bigoplus_{i \in \mathbb{Z}} A_{i}, \quad A_{i} A_{j} \subseteq A_{i+j}
$$

be a $\mathbb{Z}$-graded algebra such that there exists an integer $m$ with $A_{i}=0$ for all $i<m$ and $A_{i}$ is finite dimensional for all $i \geq m$. In this case

$$
(A \otimes A)_{k}=\bigoplus_{k=i+j} A_{i} \otimes A_{j}
$$

is finite dimentional for all $k$ and

$$
(A \otimes A)_{k}^{*}=\bigoplus_{k=i+j} \rho\left(A_{i}^{*} \otimes A_{j}^{*}\right) .
$$


The coproduct $\Delta=\rho^{-1} m^{*}$ turns the graded space

$$
\bigoplus_{i \in \mathbb{Z}} A_{i}^{*}
$$

into a coalgebra. This coalgebra is called the graded dual of the graded algebra $A$.

The following lemma is useful for studying subcoalgebras.

Lemma 2. Let $V$ be a vector space and let $W$ be a subspace of $V$. Let $a=\sum_{i=1}^{n} a_{i} \otimes b_{i}$ and let the vectors $a_{1}, \ldots, a_{n}$ be linearly independent. Assume that $a \in W \otimes W$. Then $b_{1}, b_{2}, \ldots, b_{n} \in W$.

Proof. Let $\alpha_{1}, \ldots, \alpha_{n}$ be a system of linear forms dual to $a_{1}, \ldots, a_{n}$, i.e., $\alpha_{i}\left(a_{j}\right)=\delta_{i j}$ for all $i, j$, where $\delta$ is the Kronecker delta function.

Set $\phi=\alpha_{1} \otimes$ id : $V \otimes V \mapsto V$. Then

$$
\phi\left(\sum e_{i} \otimes f_{i}\right)=\sum_{i} \alpha_{1}\left(e_{i}\right) f_{i}
$$

Obviously, $\phi(W \otimes W) \subseteq W$ and $\phi(a)=\sum_{i} \alpha_{1}\left(a_{i}\right) b_{i}=b_{1}$. Since $a \in W \otimes W$ it follows that $\phi(a) \in W$. Consequently, $b_{1} \in W$. Similarly, we get $b_{2}, \ldots, b_{n} \in W$.

\section{Examples of nON locally finite differential, Novikov, and Lie COALGEBRAS}

An algebra $A$ is called a Novikov algebra if it satisfaies the following identities:

$$
\begin{aligned}
x(y z)-(x y) z & =y(x z)-(y x) z, \\
(x y) z & =(x z) y .
\end{aligned}
$$

Recall that an algebra satisfying the identity (1) is called left-symmetric. Left-symmetric algebras are Lie-admissible, i.e., if $A$ is a left-symmetric algebra then $A$ with respect to the commutator $[x, y]:=x y-y x$ is a Lie algebra. This algebra is called the commutator algebra of $A$ and is denoted by $A^{(-)}$.

The identity (1) can be written as

$$
(x, y, z)=(y, x, z)
$$

where $(x, y, z):=(x y) z-x(y z)$ is the associator of elements $x, y, z$.

The Gelfand-Dorfman construction [5]. Let $A$ be an associative and commutative algebra with a derivation $d$. Define a new multiplication (o) on $A$ by

$$
x \circ y=x d(y),
$$

where $x, y \in A$. Then $(A, \circ)$ is a Novikov algebra.

Moreover, the vector space $A$ with respect to the bracket

$$
[x, y]=x d(y)-y d(x)
$$

is a Lie algebra. Obviously, $(A,[\cdot, \cdot])$ is the commutator algebra of the Novikov algebra $(A, \circ)$. 
Thus, any associative and commutative differential algebra $A$ generates the Novikov algebra $(A, \circ)$ and the Lie algebra $(A,[\cdot, \cdot])$. Over a field of characteristic zero every Novikov algebra can be embedded into a Novikov algebra $(A, \circ)$ for a suitable associative commutative differential algebra $A[2]$. The class of Lie algebras embeddable into Lie algebras of the type $(A,[\cdot, \cdot])$ is not described yet.

Proposition 1. A pair $(C, \Delta)$ is a Novikov coalgebra if and only if the following (co)identities hold:

$$
\begin{aligned}
(\Delta \otimes \mathrm{id}-\mathrm{id} \otimes \Delta) \Delta & =(\tau \otimes \mathrm{id})(\Delta \otimes \mathrm{id}-\mathrm{id} \otimes \Delta) \Delta, \\
(\Delta \otimes \mathrm{id}) \Delta & =(\mathrm{id} \otimes \tau)(\Delta \otimes \mathrm{id}) \Delta .
\end{aligned}
$$

Proof. Let $(C, \Delta)$ be a coalgebra and let $\alpha, \beta, \gamma \in C^{*}$ and $c \in C$. Then

$$
(\alpha, \beta, \gamma)(c)=(\alpha \otimes \beta \otimes \gamma)((\Delta \otimes \mathrm{id}-\mathrm{id} \otimes \Delta) \Delta(c))
$$

and

$$
(\beta, \alpha, \gamma)(c)=(\alpha \otimes \beta \otimes \gamma)((\tau \otimes \mathrm{id})(\Delta \otimes \mathrm{id}-\mathrm{id} \otimes \Delta) \Delta(c))
$$

Consequently,

$$
\begin{array}{r}
{[(\alpha, \beta, \gamma)-(\beta, \alpha, \gamma)](c)} \\
=(\alpha \otimes \beta \otimes \gamma)[(\Delta \otimes \mathrm{id}-\mathrm{id} \otimes \Delta) \Delta-(\tau \otimes \mathrm{id})(\Delta \otimes \mathrm{id}-\mathrm{id} \otimes \Delta) \Delta](c)) .
\end{array}
$$

This implies that the identity (3) in $C^{*}$ is equivalent to the identity (4) in $C$.

Similarly, the identity (2) in $C^{*}$ is equivalent to the identity (5) in $C$.

The Gelfand-Dorfman construction for coalgebras. Let $(C, \Delta, d)$ be an associative and commutative differential coalgebra. Define on the space $C$ a new comultiplication $\Delta_{N}$ by

$$
\Delta_{N}=(\mathrm{id} \otimes d) \Delta
$$

This means

$$
\Delta_{N}(a)=\sum_{a} a_{(1)} \otimes d\left(a_{(2)}\right)
$$

for any $a \in C$. Set also

$$
\Delta_{L}=\Delta_{N}^{(-)}=(1-\tau) \Delta_{N}
$$

i.e.,

$$
\Delta_{N}(a)=\sum_{a}\left(a_{(1)} \otimes d\left(a_{(2)}\right)-d\left(a_{(2)}\right) \otimes a_{(1)}\right)
$$

for any $a \in C$.

Proposition 2. (1) The coalgebra $\left(C, \Delta_{N}\right)$ is a Novikov coalgebra and the product in its dual algebra is defined by

$$
\alpha \circ \beta=\alpha d^{*}(\beta), \quad \alpha, \beta \in C^{*} .
$$

(2) The coalgebra $\left(C, \Delta_{L}\right)$ is a Lie coalgebra and the bracket in its dual algebra is defined by

$$
[\alpha, \beta]=\alpha d^{*}(\beta)-d_{7}^{*}(\alpha) \beta, \quad \alpha, \beta \in C^{*}
$$


Proof. By Corollary 1 , the dual $\left(C^{*}, m_{\Delta}, d^{*}\right)$ of the diferential coalgebra $(C, \Delta, d)$ is a differential algebra. We have

$$
(f g)(a)=\rho(f \otimes g)(\Delta(a))
$$

where $f, g \in C^{*}, a \in C$. By the Gelfand-Dorfman construction, the algebra $\left(C^{*}, \circ\right)$ is a Novikov algebra, where $f \circ g=f d^{*}(g)$ for all $f, g \in C^{*}$.

On the other hand,

$$
(f \circ g)(a)=\left(f d^{*}(g)\right)(a)=\sum_{a} f\left(a_{(1)}\right) d^{*}(g)\left(a_{(2)}\right)=\sum_{a} f\left(a_{(1)}\right) g\left(d\left(a_{(2)}\right)\right)=\rho(f \otimes g)\left(\Delta_{N}(a)\right),
$$
i.e.,

$$
f \circ g=f d^{*}(g)=\rho(f \otimes g) \Delta_{N}
$$

Hence $\left(C^{*}, \circ\right)$ is the dual algebra of the coalgebra $\left(C, \Delta_{N}\right)$. Since $\left(C^{*}, \circ\right)$ is a Novikov algebra it follows that $\left(C, \Delta_{N}\right)$ is a Novikov coalgebra.

The second statement of the lemma can be checked similarly.

Example 1. Let $C$ be a vector space with a linear basis

$$
e, f_{1}, f_{2}, \ldots, f_{n}, \ldots
$$

Define a comultiplication $\Delta: C \rightarrow C \otimes C$ on $C$ by

$$
\Delta(e)=e \otimes e, \Delta\left(f_{i}\right)=f_{i} \otimes e+e \otimes f_{i}, i \geq 1 .
$$

Define also a linear map $d: C \rightarrow C$ by

$$
d(e)=0, d\left(f_{i}\right)=f_{i+1}, i \geq 1 .
$$

Lemma 3. The triple $(C, \Delta, d)$ is an associative and commutative differential coalgebra.

Proof. Obviously $(C, \Delta)$ is cocommutative. Direct calculations give that

$$
\begin{array}{r}
(\Delta \otimes \mathrm{id}-\mathrm{id} \otimes \Delta) \Delta(e)=e \otimes e \otimes e-e \otimes e \otimes e=0, \\
(\Delta \otimes \mathrm{id}-\mathrm{id} \otimes \Delta) \Delta\left(f_{i}\right)=f_{i} \otimes e \otimes e+e \otimes f_{i} \otimes e \\
+e \otimes e \otimes f_{i}-f_{i} \otimes e \otimes e-e \otimes f_{i} \otimes e-e \otimes e \otimes f_{i}=0,
\end{array}
$$

i.e., $(C, \Delta)$ is coassociative.

We also have

$$
\begin{gathered}
\Delta(d(e))=0=d(e) \otimes e+e \otimes d(e) \\
\Delta\left(d\left(f_{i}\right)\right)=\Delta\left(f_{i+1}\right)=f_{i+1} \otimes e+e \otimes f_{i+1}= \\
\left(d\left(f_{i}\right) \otimes e+d(e) \otimes f_{i+1}\right)+\left(f_{i+1} \otimes d(e)+e \otimes d\left(f_{i}\right)\right), i \geq 1 .
\end{gathered}
$$

This means that $d$ is a coderivation of the coalgebra $(C, \Delta)$.

By Lemma $3,(C, \Delta, d)$ is an associative and commutative differential coalgebra. Consequently, $\left(C^{*}, m_{\Delta}, d^{*}\right)$ is an associative and commutative differential algebra. Following the tradition in the theory of ordinary differential algebras, we denote the derivative of $x \in C^{*}$ by $x^{\prime}$, i.e., $x^{\prime}=d^{*}(x)$.

Proposition 3. The differential coalgebra $(C, \Delta, d)$ is not locally finite and the differential algebra $\left(C^{*}, m_{\Delta}, d^{*}\right)$ satisfies the differential identity

$$
\begin{aligned}
& x^{\prime} y^{\prime}=0 . \\
& 8
\end{aligned}
$$


Proof. Obviously the codifferential subcoalgebra $B$ of $C$ generated by $f_{1}$ contains $f_{i}$ for all $i$. Since

$$
\Delta\left(f_{1}\right)=f_{1} \otimes e+e \otimes f_{1} \in B \otimes B
$$

it follows that $e \in B$ by Lemma 2. Therefore $B=C$ is infinite dimensional.

Let $\alpha, \beta \in C^{*}$ and $c \in C$. Then

$$
\left(d^{*}(\alpha) d^{*}(\beta)\right)(c)=\sum_{c} \alpha\left(d\left(c_{(1)}\right)\right) \beta\left(d\left(c_{(2)}\right)\right) .
$$

Consequently

$$
\left(d^{*}(\alpha) d^{*}(\beta)\right)(e)=\alpha(d(e)) \beta(d(e))=0
$$

and

$$
\left(d^{*}(\alpha) d^{*}(\beta)\right)\left(f_{i}\right)=\alpha\left(d\left(f_{i}\right)\right) \beta(d(e))+\alpha(d(e)) \beta\left(d\left(f_{i}\right)\right)=0
$$

since $d(e)=0$.

This means that $C^{*}$ satisfies the differential identity $x^{\prime} y^{\prime}=0$.

Example 2. Consider the comultiplication $\Delta_{N}=(\mathrm{id} \otimes d) \Delta$ on $C$. By Proposition 2, $\left(C, \Delta_{N}\right)$ is a Novikov coalgebra. We have

$$
\Delta_{N}(e)=(\mathrm{id} \otimes d) \Delta(e)=e \otimes d(e)=0
$$

and

$$
\Delta_{N}\left(f_{i}\right)=(\mathrm{id} \otimes d) \Delta\left(f_{i}\right)=e \otimes d\left(f_{i}\right)=e \otimes f_{i+1}
$$

for all $i \geq 1$.

Theorem 1. The Novikov coalgebra $\left(C, \Delta_{N}\right)$ is not locally finite and the Novikov algebra $C^{*}$ satisfies the identity

$$
(x y) z=0 .
$$

Proof. Let $B$ be the subcoalgebra of $\left(C, \Delta_{N}\right)$ generated by $f_{1}$. If $f_{i} \in B$ then

$$
\Delta_{N}\left(f_{i}\right)=(\mathrm{id} \otimes d) \Delta\left(f_{i}\right)=e \otimes f_{i+1} \in B \otimes B
$$

implies that $e, f_{i+1} \in B$ by Lemma 2 . Consequently, $B=C$ is infinite dimensional.

Let $\alpha, \beta, \gamma \in C^{*}$. Then

$$
\begin{gathered}
((\alpha \beta) \gamma)(e)=(\alpha \otimes \beta \otimes \gamma)(\Delta \otimes \mathrm{id}) \Delta(e)=0, \\
((\alpha \beta) \gamma)\left(f_{i}\right)=(\alpha \otimes \beta \otimes \gamma)(\Delta \otimes \mathrm{id}) \Delta\left(f_{i}\right)=(\alpha \otimes \beta \otimes \gamma)\left(\Delta(e) \otimes f_{i+1}\right)=0,
\end{gathered}
$$

since $\Delta(e)=0$. Hence the algebra $C^{*}$ satisfies the identity $(x y) z=0$.

Example 2 is the left-symmetric analogue of the non-locally finite right-symmetric coalgebra from [9].

Example 3. Now consider the Lie coalgebra $\left(C, \Delta_{L}\right)$. Recall that $\Delta_{L}=\Delta_{N}^{(-)}$and, consequently,

$$
\begin{gathered}
\Delta_{L}(e)=(d \otimes i d+i d \otimes d) \Delta(e)=0 \\
\Delta_{L}\left(f_{i}\right)=(d \otimes i d+i d \otimes d) \Delta\left(f_{i}\right)=e \otimes f_{i+1}-f_{i+1} \otimes e
\end{gathered}
$$

for all $i \geq 1$. 
Theorem 2. The Lie coalgebra $\left(C, \Delta_{L}\right)$ is not locally finite and the Lie algebra $C^{*}$ satisfies the identity

$$
[[x, y],[z, t]]=0 .
$$

Proof. Let $B$ be the subcoalgebra of $\left(C, \Delta_{L}\right)$ generated by $f_{1}$. If $f_{i} \in B$ then

$$
\Delta_{L}\left(f_{i}\right)=e \otimes f_{i+1}-f_{i+1} \otimes e \in B \otimes B
$$

implies that $e, f_{i+1} \in B$ by Lemma 2 . Consequently, $B=C$ is infinite dimensional.

Let $\alpha, \beta, \gamma, \delta \in C^{*}$. Then

$$
([\alpha, \beta])(e)=(\alpha \otimes \beta) \Delta_{L}(e)=0
$$

and

$$
\begin{array}{r}
([[\alpha, \beta],[\gamma, \delta]])\left(f_{i}\right)=(\alpha \otimes \beta \otimes \gamma \otimes \delta)\left(\Delta_{L} \otimes \Delta_{L}\right) \Delta_{L}\left(f_{i}\right) \\
=(\alpha \otimes \beta \otimes \gamma \otimes \delta)\left(\Delta_{L}(e) \otimes \Delta_{L}\left(f_{i+1}\right)-\Delta_{L}\left(f_{i+1}\right) \otimes \Delta_{L}(e)\right)=0
\end{array}
$$

since $\Delta_{L}(e)=0$. Therefore $[[\alpha, \beta],[\gamma, \delta]]=0$.

Example 3 is Michaelis's example of a non-locally finite Lie coalgebra from [11].

\section{INFINITE DIMENSIONAL COALGEBRAS WITHOUT PROPER SUBCOALGEBRAS}

Let $F$ be a field of characteristic zero and let $F[x]$ be the algebra of polynomials over $F$ in one variable $x$. Then $(F[x], m, \partial)$, where $m$ is the polynomial multiplication and $\partial=\frac{d}{d x}$, is a simple differential algebra. Consider the natural grading

$$
F[x]=F 1 \oplus F x \oplus \ldots \oplus F x^{n} \oplus \ldots
$$

The following example is the graded dual of the differential algebra $(F[x], m, \partial)$.

Example 4. Let

$$
C=F x_{0} \oplus F x_{1} \oplus \ldots \oplus F x_{n} \oplus \ldots,
$$

where $x_{i} \in F[x]^{*}$ is defined by $x_{i}\left(x^{j}\right)=\delta_{i j}$ for all $i, j$. It is easy to check that the dual comultiplication

$$
\Delta=\rho^{-1} m^{*}: C \rightarrow C \otimes C
$$

is defined by

$$
\Delta\left(x_{n}\right)=\sum_{i=0}^{n} x_{i} \otimes x_{n-i}
$$

and the coderivation

$$
d=\partial^{*}: C \rightarrow C
$$

is defined by

$$
d\left(x_{n}\right)=(n+1) x_{n+1}
$$

for all $n \geq 0$.

Consequently, $(C, \Delta, d)$ is an associative and commutative differential coalgebra.

Proposition 4. The differential coalgebra $(C, \Delta, d)$ is simple. 
Proof. Let $B$ be a nonzero subcoalgebra of $C$ and let

$$
f=x_{n}+\sum_{i<n} \alpha_{i} x_{i} \in B
$$

Then

$$
\Delta(f)=x_{n} \otimes 1+\sum_{i<n} x_{i} \otimes f_{i} \in B, \quad f_{i} \in C .
$$

By Lemma 2 this implies that $x_{n} \in B$. Since $B$ is differentially closed it follows that $x_{i} \in B$ for all $i \geq n$. Morever,

$$
\Delta\left(x_{n}\right)=\sum_{i=0}^{n} x_{i} \otimes x_{n-i} \in B
$$

imples that $x_{0}, \ldots, x_{n-1} \in B$ by Lemma 2 . Consequently, $B=C$.

Applying the Gelfand-Dorfman construction to the differential algebra $(F[x], m, \partial)$ we get a Novikov algebra $(F[x], \circ)$, where $f \circ g=f \partial(g)$. This Novikov algebra was denoted by $\mathcal{L}_{1}$ in $[10]$ and is the first algebra in the list of left-symmetric Witt algebras $\mathcal{L}_{n}$. Notice that the commutator algebra of $\mathcal{L}_{n}$ is the Witt algebra $W_{n}$ [10]. Since $\mathcal{L}_{1}$ is a Novikov algebra, we call $\mathcal{L}_{1}$ the Novikov-Witt algebra. Recall that

$$
\mathcal{L}_{1}=F \partial \oplus F x \partial \oplus \ldots \oplus F x^{n} \partial \oplus \ldots
$$

is the algebra of all derivations of $F[x]$ with respect to the product

$$
x^{i} \partial \circ x^{j} \partial=j x^{i+j-1} \partial, \quad i, j \geq 0 .
$$

The following example is the graded dual of the Novikov-Witt algebra $\mathcal{L}_{1}$.

Example 5. Let $(C, \Delta, d)$ be the differential coalgebra from Example 4. Applying the Gelfand-Dorfman construction, we get a Novikov coalgebra $\left(C, \Delta_{N}\right)$, where

$$
\Delta_{N}=(\mathrm{id} \otimes d) \Delta
$$

i.e.,

$$
\Delta_{N}\left(x_{n}\right)=\sum_{i=0}^{n}(n-i+1) x_{i} \otimes x_{n-i+1}
$$

Theorem 3. The Novikov coalgebra $\left(C, \Delta_{N}\right)$ is simple.

Proof. Let $B$ be a nonzero subcoalgebra of $C$ and let

$$
f=x_{n}+\sum_{i<n} \alpha_{i} x_{i} \in B
$$

Then

$$
\Delta_{N}(f)=(n+1) 1 \otimes x_{n+1}+\sum_{\substack{i<n+1 \\ 11}} f_{i} \otimes x_{i} \in B, \quad f_{i} \in C
$$


By Lemma 2 this implies that $x_{n+1} \in B$. Consequently, we can assume that $x_{n} \in B$ for some $n \geq 0$. Then

$$
\Delta_{N}\left(x_{n}\right)=\sum_{i=0}^{n}(n-i+1) x_{i} \otimes x_{n-i+1} \in B
$$

implies that $x_{0}, \ldots, x_{n+1} \in B$ by Lemma 2 . Consequently, $B=C$.

The following example is the graded dual of the Witt algebra $W_{1}$.

Example 6. Let $(C, \Delta, d)$ be the Novikov coalgebra from Example 5 . Then the commutator coalgebra $\left(C, \Delta_{L}\right)$ is a Lie coalgebra, where

$$
\Delta_{L}=\Delta_{N}^{(-)}=(1-\tau) \Delta_{N}
$$

i.e.,

$$
\begin{array}{r}
\Delta_{L}\left(x_{n}\right)=\sum_{i=0}^{n}(n-i+1) x_{i} \otimes x_{n-i+1}-(i+1) x_{i+1} \otimes x_{n-i} \\
=\sum_{i=0}^{n+1}(n+1-2 i) x_{i} \otimes x_{n+1-i} .
\end{array}
$$

Theorem 4. The Lie coalgebra $\left(C, \Delta_{L}\right)$ is simple.

Proof. Let $B$ be a nonzero subcoalgebra of $C$ and let

$$
f=x_{n}+\sum_{i<n} \alpha_{i} x_{i} \in B
$$

Then, as in the proof of Theorem 3, we get $x_{n+1} \in B$. Applying Lemma 2 to the inclusion $\Delta_{L}\left(x_{n}\right) \in B \otimes B$, we get $x_{0} \in B$. Consequently, $B=C$.

Notice that an example of an infinite dimensional Lie coalgebra without finite dimensional subcoagebras was constructed in [12] and [13].

\section{Non locally finite Jordan supecoalgebras}

Let $G$ be the Grassman algebra with identity. Then $G=G_{0}+G_{1}$ is a $Z_{2}$-graded algebra. Let $J=J_{0}+J_{1}$ be a $Z_{2}$-graded algebra. Then $G(J)=J_{0} \otimes G_{0}+J_{1} \otimes G_{1}$ is a subalgebra of the algebra $G \otimes J$. The subalgebra $G(J)$ is called Grassman envelope of the algebra $J$.

An algebra $J$ is called a Jordan superalgebra, if its Grassman envelope $G(J)$ is a Jordan algebra, i.e., $G(J)$ satisfies the following identities:

$$
\begin{aligned}
x y & =y x, \\
\left(x^{2} y\right) x & =x^{2}(y x) .
\end{aligned}
$$

The Kantor construction [8]. Let $A$ be an associative commutative algebra over $F$ with a derivation $D$. Denote by $\bar{A}$ an isomorphic copy of the vector space $A$ with an isomorphism $a \mapsto \bar{a}$. On the direct sum of the vector spaces

$$
J(A, D)=A \oplus \bar{A}
$$


define a product $(\cdot)$ by

$$
a \cdot b=a b, a \cdot \bar{b}=\overline{a b}, \bar{a} \cdot b=\overline{a b}, \bar{a} \cdot \bar{b}=a D(b)-D(a) b,
$$

where $a, b \in A$ and $a b$ is the product of elements in $A$. Then $J(A, D)$ is a Jordan superalgebra. The superalgebra $J(A, D)$ is called a superalgebra of the vector type.

The Kantor construction for coalgebras. Let $(C, \Delta, d)$ be an associtive and commutative differential coalgebra. Let $\bar{C}$ be an isomorphic copy of the vector space $C$ with an isomorphism $c \mapsto \bar{c}$. On the direct sum of vector spaces

$$
J(C, d)=C \oplus \bar{C}
$$

define a coproduct $\Delta_{J}$ by

$$
\begin{array}{r}
\Delta_{J}(c)=\sum_{(c)} c_{(1)} \otimes c_{(2)}+\overline{c_{(1)}} \otimes \overline{d\left(c_{(2)}\right)}-\overline{d\left(c_{(1)}\right)} \otimes \overline{c_{(2)}}, \\
\Delta_{J}(\bar{c})=\sum_{(c)} \overline{c_{(1)}} \otimes c_{(2)}+c_{(1)} \otimes \overline{c_{(2)}},
\end{array}
$$

where $c \in C$ and $\Delta(c)=\sum_{(c)} c_{(1)} \otimes c_{(2)}$.

Proposition 5. The coalgebra $\left(J(C, d), \Delta_{J}\right)$ is a Jordan supercoalgebra and its dual $J\left(C^{*}, d^{*}\right)$ is a Jordan superalgebra of the vector type.

Proof. By Corollary $1,\left(C^{*}, m_{\Delta}, d^{*}\right)$ is an associative and commutative differential algebra. We have $J(C, d)^{*}=C^{*}+(\bar{C})^{*}$. The isomorphism of $C$ and $\bar{C}$ induces the isomorphism of $C^{*}$ and $(\bar{C})^{*}$. Under this isomorphism, for any $\alpha \in C^{*}$ there corresponds $\bar{\alpha} \in(\bar{C})^{*}$ such that $\alpha(c)=\bar{\alpha}(\bar{c})$ for any $c \in C$. Therefore, we can write that $J(C, d)^{*}=C^{*}+\overline{C^{*}}$.

Let $\alpha, \beta \in C^{*}$, and $c \in C$. Denote by $(\cdot)$ the multiplication of the algebra $\left(J(C, d), \Delta_{J}\right)^{*}$. Then we have

$$
\begin{aligned}
& (\alpha \cdot \beta)(c)=\rho(\alpha \otimes \beta) \Delta_{J}(c),(\alpha \cdot \bar{\beta})(\bar{c})=\rho(\alpha \otimes \bar{\beta}) \Delta_{J}(\bar{c}), \\
& (\bar{\alpha} \cdot \beta)(\bar{c})=\rho(\bar{\alpha} \otimes \beta) \Delta_{J}(\bar{c}),(\bar{\alpha} \cdot \bar{\beta})(c)=\rho(\bar{\alpha} \otimes \bar{\beta}) \Delta_{J}(c) .
\end{aligned}
$$

From this we get

$$
\alpha \cdot \beta=\alpha \beta, \alpha \cdot \bar{\beta}=\overline{\alpha \beta}, \bar{\alpha} \beta=\overline{\alpha \beta}, \bar{\alpha} \cdot \bar{\beta}=\alpha d^{*}(\beta)-d^{*}(\alpha) \beta,
$$

where $\alpha \beta$ is the product of elements in the dual algebra $\left(C^{*}, m_{\Delta}, d^{*}\right)$.

Consequently, $\left(J(C, d), \Delta_{J}\right)^{*}=J\left(C^{*}, d^{*}\right)$.

Example 7. Let $(C, \Delta, d)$ be the differential coalgebra from Example 1 and let $\left(J(C, d), \Delta_{J}\right)$ be the Jordan supercoalgebra obtained from $(C, \Delta, d)$ by the Kantor construction for coalgebras. Notice that

$$
\begin{gathered}
\Delta_{J}(e)=e \otimes e, \quad \Delta_{J}\left(f_{i}\right)=e \otimes f_{i}+f_{i} \otimes e+\bar{e} \otimes \overline{f_{i+1}}-\overline{f_{i+1}} \otimes \bar{e}, \\
\Delta_{J}(\bar{e})=e \otimes \bar{e}+\bar{e} \otimes e, \quad \Delta_{J}\left(\overline{f_{i}}\right)=e \otimes \overline{f_{i}}+\overline{f_{i}} \otimes e+\bar{e} \otimes f_{i}+f_{i} \otimes \bar{e},
\end{gathered}
$$

for all $i \geq 1$. 
Theorem 5. The Jordan super-coalgebra $\left(J(C, d), \Delta_{J}\right)$ is not locally finite and its dual superalgebra satisfies the super identities

$$
x y=y x, x z=z x,\left(z_{1} z_{2}\right)\left(z_{3} z_{4}\right)=0
$$

for all even $x, y$ and odd $z, z_{1}, z_{2}, z_{3}, z_{4}$.

Proof. Let $B$ be the subcoalgebra of $J(C, d)$ generated by $\overline{f_{1}}$. If $\overline{f_{i}} \in B$, then $\Delta_{J}\left(\overline{f_{i}}\right) \in$ $B \otimes B$ implies that $e, \bar{e}, f_{i} \in B$ by Lemma 2. If $f_{i} \in B$, then $\Delta_{J}\left(f_{i}\right) \in B \otimes B$ implies that $\overline{f_{i+1}} \in B$. Consequently, $B=J(C, d)$ is infinite dimensional.

By Proposition 5 , the dual of the supercoalgebra $\left(J(C, d), \Delta_{J}\right)$ is the Jordan superalgebra $J\left(C^{*}, d^{*}\right)$ obtained from the differential algebra $\left(C^{*}, m_{\Delta}, d^{*}\right)$ by the Kantor construction. The first two identities of $J\left(C^{*}, d^{*}\right)$, mentioned in the lemma, directly follow from the Kantor construction since $C^{*}$ is an associative and commutative algebra. Notice that the product of two odd elements $z_{1}, z_{2}$ from $J\left(C^{*}, d^{*}\right)$ belongs to the ideal $d^{*}\left(C^{*}\right) C^{*}$. By Proposition 3 , we have $d^{*}\left(C^{*}\right)^{2}=0$. Consequently, $\left(z_{1} z_{2}\right)\left(z_{3} z_{4}\right)=0$ for all odd elements $z_{1}, z_{2}, z_{3}, z_{4}$.

The following example is the graded dual of the simple Jordan superalgebra $J(F[x], \partial)$ obtained from the simple differential algebra $(F[x], \partial)$ by the Kantor construction.

Example 8. Let $(C, \Delta, d)$ be the codifferential coalgebra from Example 4 and let $\left(J(C, d), \Delta_{J}\right)$ be the Jordan super-coalgebra obtained from $(C, \Delta, d)$ by the Kantor construction for coalgebras. Direct calculations give that

$$
\begin{gathered}
\Delta_{J}\left(x_{n}\right)=\sum_{i=0}^{n} x_{i} \otimes x_{n-i}+\sum_{i=0}^{n+1}(n+1-2 i) \bar{x}_{i} \otimes \bar{x}_{n-i+1}, \\
\Delta_{J}\left(\bar{x}_{n}\right)=\sum_{i=0}^{n}\left(\bar{x}_{i} \otimes x_{n-i}+x_{i} \otimes \bar{x}_{n-i}\right),
\end{gathered}
$$

for all $n \geq 0$.

Theorem 6. The Jordan supercoalgebra $\left(J(C, d), \Delta_{J}\right)$ is simple.

Proof. Let $B$ be a nonzero subcoalgebra of $\left(J(C, d), \Delta_{J}\right)$ and let

$$
f=x_{n}+\sum_{i<n} \alpha_{i} x_{i}+\bar{c} \in B
$$

where $\bar{c} \in \bar{C}$. Then

$$
\Delta_{J}(f)=\sum_{i \geq 0} a_{i} \otimes x_{i}+\sum_{i \geq 0} b_{i} \otimes \bar{x}_{i} \in B \otimes B
$$

and it is easy to check that $b_{n+1} \neq 0$. By Lemma $2, \bar{x}_{n+1} \in B$. Then $\Delta_{J}\left(\bar{x}_{n+1}\right) \in B \otimes B$ implies that $x_{0}, \ldots, x_{n+1}, \bar{x}_{0}, \ldots, \bar{x}_{n+1} \in B$. Consequently, $B=C$.

If

$$
f=\bar{x}_{n}+\sum_{i<n} \alpha_{i} \bar{x}_{i} \in B
$$

then $\Delta_{J}(f) \in B \otimes B$ implies that $x_{n}, \bar{x}_{n} \in B$ by Lemma 2. Continuing the same discussions, we get $B=C$. 
Notice that an example of a Jordan super-coalgebra without finite dimensional subcoagebras was constructed in [23]

\section{Non LOCALly finite Right Alternative COALGEBRA}

In this section we give a corrected version of the example of a non locally finite rightalternative coalgebra from [9]. This example was constructed on the base of the example of a finitely generated metabelian right-alternative algebra that is not residually finite [19].

An algebra $A$ is called right-alternative if it satisfies the following identities:

$$
\begin{gathered}
(y x) x=y x^{2}, \\
((x y) z) y=x((y z) y) .
\end{gathered}
$$

The identity (6) is called right-alternativity and can be written in terms of the associators as

$$
(y, x, x)=0 .
$$

Over fields of characteristic $\neq 2$ this identity also implies the Moufang identity (7) (see $[25])$.

Proposition 6. Let $(A, \Delta)$ be a coalgebra over a field $F$ of characteristic $\neq 2$. The coalgebra $(A, \Delta)$ is right-alternative if and only if the following identity holds:

$$
(\Delta \otimes \mathrm{id}-\mathrm{id} \otimes \Delta) \Delta+(\mathrm{id} \otimes \tau)(\Delta \otimes \mathrm{id}-\mathrm{id} \otimes \Delta) \Delta=0 .
$$

Proof. Let $(C, \Delta)$ be a coalgebra and let $\alpha, \beta, \gamma \in C^{*}$ and $c \in C$. Then

$$
(\alpha, \beta, \gamma)(c)=(\alpha \otimes \beta \otimes \gamma)((\Delta \otimes \mathrm{id}-\mathrm{id} \otimes \Delta) \Delta(c))
$$

and

$$
(\alpha, \gamma, \beta)(c)=(\alpha \otimes \beta \otimes \gamma)((\mathrm{id} \otimes \tau)(\Delta \otimes \mathrm{id}-\mathrm{id} \otimes \Delta) \Delta(c)) .
$$

Consequently,

$$
\begin{array}{r}
{[(\alpha, \beta, \gamma)+(\alpha, \gamma, \beta)](c)} \\
=(\alpha \otimes \beta \otimes \gamma)[(\Delta \otimes \mathrm{id}-\mathrm{id} \otimes \Delta) \Delta+(\mathrm{id} \otimes \tau)(\Delta \otimes \mathrm{id}-\mathrm{id} \otimes \Delta) \Delta](c) .
\end{array}
$$

This implies that the identity (9) in $C$ is equivalent to linearized version of the identity (8) in $C^{*}$. Consequently, (9) is equivalent to (8) over fields of characteristic $\neq 2$.

Example 9. Let $A$ be a vector space with a linear basis

$$
e_{1}, e_{2}, f_{1}, f_{2}, \ldots, f_{n}, \ldots
$$

Define a comultiplication $\Delta$ on the vector space $A$ by

$$
\begin{gathered}
\Delta\left(e_{1}\right)=0, \Delta\left(e_{2}\right)=0, \\
\Delta\left(f_{3 n-2}\right)=e_{1} \otimes f_{3 n}, \Delta\left(f_{3 n-1}\right)=e_{2} \otimes f_{3 n}, \\
\Delta\left(f_{3 n}\right)=e_{2} \otimes f_{3 n+1}-f_{3 n+1} \otimes e_{2}-e_{1} \otimes f_{3 n+2}+f_{3 n+2} \otimes e_{1}, n \geq 1 . \\
15
\end{gathered}
$$


Theorem 7. The coalgebra $(A, \Delta)$ is a right-alternative non locally finite coalgebra. Moreover, the dual algebra $A^{*}$ satisfies the identities $(x y)(z t)=((x y) z) t=0$.

Proof. Let $\Delta_{A s s}=(\Delta \otimes$ id - id $\otimes \Delta) \Delta$. If $f, g, h \in A^{*}$, then

$$
(f, g, h)(a)=(f \otimes g \otimes h)\left(\Delta_{a s s}(a)\right)
$$

for all $a \in A$. We have $(f, g, g)\left(e_{i}\right)=0$ since $\Delta\left(e_{i}\right)=0$ for $i=1,2$.

Direct calculations give that

$$
\begin{gathered}
\Delta_{A s s}\left(f_{3 n-2}\right)=(\Delta \otimes \mathrm{id}-\mathrm{id} \otimes \Delta)\left(e_{1} \otimes f_{3 n}\right)=-e_{1} \otimes \Delta\left(f_{3 n}\right) \\
=-e_{1} \otimes e_{2} \otimes f_{3 n+1}+e_{1} \otimes f_{3 n+1} \otimes e_{2}+e_{1} \otimes e_{1} \otimes f_{3 n+2}-e_{1} \otimes f_{3 n+2} \otimes e_{1}, \\
\Delta_{A s s}\left(f_{3 n-1}\right)=-e_{2} \otimes \Delta\left(f_{3 n}\right)=-e_{2} \otimes e_{2} \otimes f_{3 n+1} \\
+e_{2} \otimes f_{3 n+1} \otimes e_{2}+e_{2} \otimes e_{1} \otimes f_{3 n+2}-e_{2} \otimes f_{3 n+2} \otimes e_{1},
\end{gathered}
$$

and

$$
\begin{array}{r}
\Delta_{A s s}\left(f_{3 n}\right)=(\Delta \otimes \mathrm{id}-\mathrm{id} \otimes \Delta)\left(e_{2} \otimes f_{3 n+1}-f_{3 n+1} \otimes e_{2}-e_{1} \otimes f_{3 n+2}+f_{3 n+2} \otimes e_{1}\right) \\
=-\Delta\left(f_{3 n+1}\right) \otimes e_{2}+\Delta\left(f_{3 n+2}\right) \otimes e_{1}-e_{2} \otimes \Delta\left(f_{3 n+1}\right)+e_{1} \otimes \Delta\left(f_{3 n+2}\right) \\
=-e_{1} \otimes f_{3(n+1)} \otimes e_{2}+e_{2} \otimes f_{3(n+1)} \otimes e_{1}-e_{2} \otimes e_{1} \otimes f_{3(n+1)}+e_{1} \otimes e_{2} \otimes f_{3(n+1)} .
\end{array}
$$

Consequently,

$$
\begin{array}{r}
(f, g, g)\left(f_{3 n-2}\right)=-f\left(e_{1}\right) g\left(e_{2}\right) g\left(f_{3 n+1}\right)+f\left(e_{1}\right) g\left(f_{3 n+1}\right) g\left(e_{2}\right) \\
+f\left(e_{1}\right) g\left(e_{1}\right) g\left(f_{3 n+2}\right)-f\left(e_{1}\right) g\left(f_{3 n+2}\right) g\left(e_{1}\right)=0 \\
(f, g, g)\left(f_{3 n-1}\right)=-f\left(e_{2}\right) g\left(e_{2}\right) g\left(f_{3 n+1}\right)+f\left(e_{2}\right) g\left(f_{3 n+1}\right) g\left(e_{2}\right) \\
+f\left(e_{2}\right) g\left(e_{1}\right) g\left(f_{3 n+2}\right)-f\left(e_{2}\right) g\left(f_{3 n+2}\right) g\left(e_{1}\right)=0
\end{array}
$$

and

$$
\begin{array}{r}
(f, g, g)\left(f_{3 n}\right)=-f\left(e_{1}\right) g\left(f_{3(n+1)}\right) g\left(e_{2}\right)+f\left(e_{2}\right) g\left(f_{3(n+1)}\right) g\left(e_{1}\right) \\
-f\left(e_{2}\right) g\left(e_{1}\right) g\left(f_{3(n+1)}\right)+f\left(e_{1}\right) g\left(e_{2}\right) g\left(f_{3(n+1)}\right)=0 .
\end{array}
$$

Consequently, $(f, g, g)(a)=0$ for all $f, g \in A^{*}$ and $a \in A$, i.e., $(f, g, g)=0$. This means that $A^{*}$ satisfies the identity (8).

Set $\phi=(\Delta \otimes \mathrm{id} \otimes \mathrm{id})(\Delta \otimes \mathrm{id}) \Delta$. Direct calculations give that

$$
\phi\left(e_{1}\right)=\phi\left(e_{2}\right)=0,
$$

$\phi\left(f_{3 n-2}\right)=(\Delta \otimes \mathrm{id} \otimes \mathrm{id})(\Delta \otimes \mathrm{id})\left(e_{1} \otimes f_{3 n}\right)=(\Delta \otimes \mathrm{id} \otimes \mathrm{id})\left(\Delta\left(e_{1}\right) \otimes f_{3 n}\right)=0$,

$\phi\left(f_{3 n-1}\right)=(\Delta \otimes \mathrm{id} \otimes \mathrm{id})(\Delta \otimes \mathrm{id})\left(e_{2} \otimes f_{3 n}\right)=(\Delta \otimes \mathrm{id} \otimes \mathrm{id})\left(\Delta\left(e_{2}\right) \otimes f_{3 n}\right)=0$,

and

$$
\begin{array}{r}
\phi\left(f_{3 n}\right)=(\Delta \otimes \mathrm{id} \otimes \mathrm{id})\left(-\Delta\left(f_{3 n+1}\right) \otimes e_{2}+\Delta\left(f_{3 n+2}\right) \otimes e_{1}\right)= \\
(\Delta \otimes \mathrm{id} \otimes \mathrm{id})\left(-e_{1} \otimes f_{3(n+1)} \otimes e_{2}+e_{2} \otimes f_{3(n+1)} \otimes e_{1}\right)=0
\end{array}
$$

for all $n \geq 1$.

Consequently, $(((f g) h) e)(a)=0$ for all $f, g, h, e \in A^{*}$ and $a \in A$, i. e., $((f g) h) e=0$. This means that $A^{*}$ satisfies the identity $((x y) z) t=0$. 
Now set $\psi=(\mathrm{id} \otimes \Delta \otimes \mathrm{id})(\mathrm{id} \otimes \Delta) \Delta$. Then

$$
\begin{array}{r}
\psi\left(e_{1}\right)=\psi\left(e_{2}\right)=0, \\
\begin{array}{r}
\psi\left(f_{3 n-2}\right)=(\mathrm{id} \otimes \Delta \otimes \mathrm{id})(\mathrm{id} \otimes \Delta)\left(e_{1} \otimes f_{3 n}\right)=(\mathrm{id} \otimes \Delta \otimes \mathrm{id})\left(e_{1} \otimes \Delta\left(f_{3 n}\right)\right) \\
=-e_{1} \otimes \Delta\left(f_{3 n+1}\right) \otimes e_{2}+e_{1} \otimes \Delta\left(f_{3 n+2}\right) \otimes e_{1} \\
=-e_{1} \otimes e_{1} \otimes f_{3(n+1)} \otimes e_{2}+e_{1} \otimes e_{2} \otimes f_{3(n+1)} \otimes e_{1}, \\
\psi\left(f_{3 n-1}\right)=(\mathrm{id} \otimes \Delta \otimes \mathrm{id})(\mathrm{id} \otimes \Delta)\left(e_{2} \otimes f_{3 n}\right)=(\mathrm{id} \otimes \Delta \otimes \mathrm{id})\left(e_{2} \otimes \Delta\left(f_{3 n}\right)\right) \\
=-e_{2} \otimes \Delta\left(f_{3 n+1}\right) \otimes e_{2}+e_{2} \otimes \Delta\left(f_{3 n+2}\right) \otimes e_{1} \\
=-e_{2} \otimes e_{1} \otimes f_{3(n+1)} \otimes e_{2}+e_{2} \otimes e_{2} \otimes f_{3(n+1)} \otimes e_{1},
\end{array}
\end{array}
$$

and

$$
\begin{array}{r}
\psi\left(f_{3 n}\right)=(\mathrm{id} \otimes \Delta \otimes \mathrm{id})(\mathrm{id} \otimes \Delta)\left(e_{2} \otimes f_{3 n+1}-f_{3 n+1} \otimes e_{2}-e_{1} \otimes f_{3 n+2}+f_{3 n+2} \otimes e_{1}\right) \\
=(\mathrm{id} \otimes \Delta \otimes \mathrm{id})\left(e_{2} \otimes \Delta\left(f_{3 n+1}\right)-e_{1} \otimes \Delta\left(f_{3 n+2}\right)\right) \\
=(\mathrm{id} \otimes \Delta \otimes \mathrm{id})\left(e_{2} \otimes e_{1} \otimes f_{3(n+1)}-e_{1} \otimes e_{2} \otimes f_{3(n+1)}\right) \\
=e_{2} \otimes \Delta\left(e_{1}\right) \otimes f_{3(n+1)}-e_{1} \otimes \Delta\left(e_{2}\right) \otimes f_{3(n+1)}=0
\end{array}
$$

for all $n \geq 1$.

Therefore,

$$
\begin{gathered}
(f((g h) g))\left(f_{3 n-2}\right)=-f\left(e_{1}\right) g\left(e_{1}\right) h\left(f_{3(n+1)}\right) g\left(e_{2}\right)+f\left(e_{1}\right) g\left(e_{2}\right) h\left(f_{3(n+1)}\right) g\left(e_{1}\right)=0 \\
(f((g h) g))\left(f_{3 n-1}\right)=0
\end{gathered}
$$

for all $f, g, h \in A^{*}$.

This means $f((g h) g)=0$ for all $f, g, h \in A^{*}$. Together with the identity $((x y) z) t=0$, this proves that the Moufang identity (7) holds in $A^{*}$.

It is easy to check that $(\Delta \otimes \Delta) \Delta(a)=0$ for all $a \in A$. Therefore $A^{*}$ satisfies the identity $(x y)(z t)=0$.

Now we show that $(A, \Delta)$ is not locally finite. Let $B$ a subalgebra of $(A, \Delta)$ generated by $f_{1}, f_{2}$.

Suppose that $f_{i} \in B$. If $i=3 n-1$ or $i=3 n-2$, then $\Delta\left(f_{i}\right) \in B \otimes B$ implies that $e_{1}, e_{2}, f_{3 n} \in B$ by Lemma 2 . If $i=3 n$, then we get $e_{1}, e_{2}, f_{3 n+1}, f_{3 n+2} \in B$. This implies that $B=C$ is infinite-dimensional.

\section{Acknowledgments}

The first author is supported by the grant of the Ministry of Education and Science of the Republic of Kazakhstan (project AP09261086) and the other two authors are supported by the Russian Science Foundation (project 21-11-00286). 


\section{REFERENCES}

[1] J. Anquela, T. Cortes, F. Montaner, Nonassociative coalgebras. Comm. Algebra 22 (1994), no. 12, $4693-4716$.

[2] L.A. Bokut, Y. Chen, Z. Zhang, Gröbner-Shirshov bases method for Gelfand-Dorfman-Novikov algebras. J. Algebra Appl. 16 (2017), no. 1, 1750001, 22 pp.

[3] V.G. Drinfeld, Quantum groups. Proceedings of the International Congress of Mathematicians, Vol. 1, 2 (Berkeley, Calif., 1986), 798-820, Amer. Math. Soc., Providence, RI, 1987.

[4] V.G. Drinfeld, Hamiltonian structures on Lie groups, Lie bialgebras and the geometric meaning of classical Yang-Baxter equations. (Russian) Dokl. Akad. Nauk SSSR 268 (1983), no. 2, 285-287.

[5] I.M. Gelfand, I.Ya. Dorfman, Hamiltonian operators and algebraic structures associated with them. (Russian) Funktsional. Anal. i Prilozhen. 13 (1979), no. 4, 13-30

[6] M.E. Goncharov, V.N. Zhelyabin, Mikheev's construction for Mal'tsev coalgebras. (Russian) Algebra Logika 51 (2012), no. 5, 668-671; translation in Algebra Logic 51 (2012), no. 5, 445-447.

[7] M.E. Goncharov, V.N. Zhelyabin, Embedding of Mal'tsev coalgebras into Lie coalgebras with triality. (Russian) Algebra Logika 52 (2013), no. 1, 34-56; translation in Algebra Logic 52 (2013), no. 1, 2440.

[8] I.L. Kantor, Jordan and Lie superalgebras determined by a Poisson algebra. Algebra and Analysis (Tomsk, 1989), 55-80, Amer. Math. Soc. Transl. Ser. 2, 151, Amer. Math. Soc., Providence, RI, 1992.

[9] D.Kh. Kozybaev, Right-alterenative and right-symmertric coalgebras, South-Kazakhstan Science and Education. 19 (2000), No. 12, 155-163.

[10] D. Kozybaev, U. Umirbaev, Identities of the left-symmetric Witt algebras. Internat. J. Algebra Comput. 26 (2016), no. 2, 435-450.

[11] W. Michaelis, Lie coalgebras. Adv. in Math. 38 (1980), no. 1, 1-54.

[12] W. Michaelis, An example of a non-zero Lie coalgebra $M$ for which $\operatorname{Loc}(M)=0$. J. Pure Appl. Algebra 68 (1990), no. 3, 341-348.

[13] W.D. Nichols, The structure of the dual Lie coalgebra of the Witt algebra. J. Pure Appl. Algebra 68 (1990), no. 3, 359-364.

[14] D.E. Radford, Hopf algebras. Series on Knots and Everything, 49. World Scientific Publishing Co. Pte. Ltd., Hackensack, NJ, 2012. xxii+559 pp.

[15] G. Santos Filho, L. Murakami, I. Shestakov, Locally finite coalgebras and the locally nilpotent radical I. Linear Algebra Appl. 621 (2021), 235-253.

[16] G. Santos Filho, L. Murakami, I. Shestakov. Locally finite coalgebras and the locally nilpotent radical II. Comm. Algebra 49 (2021), no. 12, 5472-5482.

[17] A.M. Slinko, Local finiteness of coalgebraic Lie coalgebras. Comm. Algebra 23 (1995), no. 3, 11651170 .

[18] M. Sweedler, Hopf algebras. Mathematics Lecture Note Series W. A. Benjamin, Inc., New York 1969 vii $+336 \mathrm{pp}$.

[19] U.U. Umirbaev, The word problem for metabelian right-alternative algebras. (Russian) Modeltheoretic algebra, 130-139, Kazakh. Gos. Univ., Alma Ata, 1989.

[20] V.N. Zhelyabin, Structurable coalgebras. (Russian) Algebra i Logika 35 (1996), no. 5, 529-542; translation in Algebra and Logic 35 (1996), no. 5, 296-304.

[21] V.N. Zhelyabin, The Kantor-Koecher-Tits construction for Jordan coalgebras. (Russian) Algebra i Logika 35 (1996), no. 2, 173-189; translation in Algebra and Logic 35 (1996), no. 2, 96-104.

[22] V.N. Zhelyabin, Jordan (super)coalgebras and Lie (super)coalgebras. (Russian) Sibirsk. Mat. Zh. 44 (2003), no. 1, 87-111; translation in Siberian Math. J. 44 (2003), no. 1, 73-92.

[23] V.N. Zhelyabin, Dual coalgebras of Jordan bialgebras and superalgebras. (Russian) Sibirsk. Mat. Zh. 46 (2005), no. 6, 1302-1315; translation in Siberian Math. J. 46 (2005), no. 6, 1050-1061.

[24] V.N. Zhelyabin, Embedding of Jordan copairs into Lie coalgebras. Comm. Algebra 35 (2007), no. 2, $561-576$. 
[25] K.A. Zhevlakov, A. M. Slinko, I. P. Shestakov, A. I. Shirshov, Rings that are nearly associative. Pure and Applied Mathematics, 104. Academic Press, New York-London, 1982. xi+371 pp 